\title{
PROSTATE BIOPSY UP DATE
}

\author{
SALAM MA ${ }^{1}$, KAISER $I^{2}$, ISLAMGS ${ }^{3}$, MAMOON I ${ }^{3}$
}

\begin{abstract}
The prostate biopsies are usually performed by urologists in the office setting using trans rectal ultrasound (TRUS) guidance. Current standard of care involves obtaining usually 12 cores from different anatomical sections. These biopsies are usually not directed into a specific lesion as most prostate cancers are not visible on TRUS. ColorDoppler, ultrasound contrast agents, elastography, MRI, and MRI/ultrasound fusion are proposed as imaging methods to guide prostate biopsies. Prostate MRI and fusion biopsy create opportunities for diagnostic and interventional radiologists to play an increasingly important role in the screening, evaluation, diagnosis, targeted biopsy, surveillance and focal therapy of the prostate cancer patient.
\end{abstract}

Bangladesh J. Urol. 2018; 21(2): 145-157

\section{Introduction}

The indications for prostate biopsy include a positive digital rectal exam (focal nodule, stiffness, or asymmetry), clinical symptoms, high serum prostate specific antigen (PSA) or PSA velocity. The Prostate Biopsy is also indicated to monitor in known prostate cancer patients who are on active surveillance. The standard of care involves obtaining $10-14$ cores from different anatomical sections of the prostate. Ultrasound has low sensitivity and is limited by significant overlap in the appearances of benign changes and malignancy [1-3]. In PSA screening has been the primary determinant for prostate biopsies in the general population but has resulted in over-diagnosis and overtreatment, without a definite survival benefit[2-6]. In the United States the Increased incidence of Biopsy in PSA screening program has been called into question by the United States Preventive The Task Force[7]. To day in most nation PSA screening is performed on an individualized basis after discussion of the risks and benefits of screening.

Before the imaging methods, the prostate biopsy was guided by direct palpation. Use of TRUS began in the

1. Professor of Urooncology, and Ex Chairman Urology, BSMMU, Dhaka

2. Consultant, Urology and Transplantation Foundation of Bangladesh, 57/9 Panthapath Avenue, Dhaka 1215

3. Urology and Transplantation Foundation of Bangladesh, 57/9 Panthapath Avenue, Dhaka 1215

Correspondences: Dr. Salam MA, Urology and Transplantation Foundation of Bangladesh, 57/9, Panthapath Avenue, Dhaka 1215 Received: 9 August 2017 early 1970s with advent of ultrasound, and the original sextant biopsy scheme (total of six cores from the base, middle, and apex bilaterally) improved detection over digital guidance[8]. Later multiple studies showed that doubling the number of cores was associated with improved cancer detection by $31 \%[9]$. As a result, $12-$ 18 core systematic biopsy became the standard in the 2000s. Increase in biopsy cores from six to twelve is not associated with measurable increased post-biopsy morbidity[10]. Saturation biopsy, which involves sampling the entire gland but is reserved for patients with persistently rising PSA and a history of negative biopsies[11].

To provide better image guidance of prostate biopsies, a number of ultrasound-based technologies were introduced. These included Doppler-targeted strategies, real-time elastography, and ultrasound contrast agents. Other ultrasound techniques include 3D ultrasound and tissue characterization algorithms [9].

The MRI of the prostate appears to be the most sensitive method for detecting prostate cancer by imaging. Direct biopsies under MR guidance have been attempted but prove to be inferior. Better cancer detection rates were demonstrated when the pre-biopsy MRI was fused to a real-time TRUS to guide biopsy to lesions seen on MRI[12]. The techniques for fusion guidance include electromagnetic tracking, optical tracking and encoded mechanical arm has been evolved and currently is in practice in most advanced centers. 


\section{Applied Prostate anatomy and zonal distribution}

Prostate gland is comprised of peripheral, transitional, and central zones. Peripheral zone is disc-shaped and constitutes $70 \%$ of the prostate gland. Its ducts radiate laterally from the urethra lateral and distal to the verumontanum[13]. Central zone constitutes $25 \%$ of the prostate gland, and surrounds the prostatic urethra. Its ducts arise close to the ejaculatory duct orifices at the verumontanum and branch laterally near the prostate base.

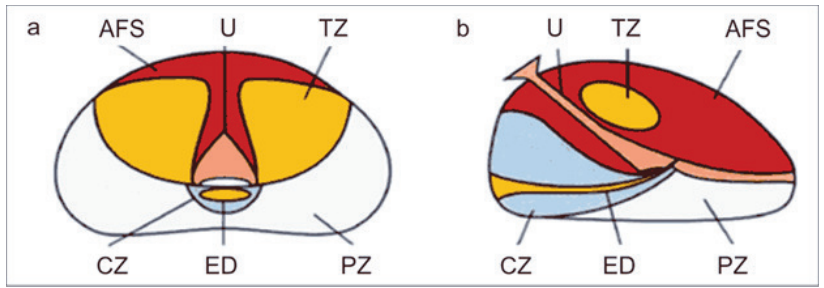

Fig. 1. McNeal's zonal anatomy of the prostate: a transverse section, $b$ - longitudinal section

$\mathrm{TZ}$ - transition zone; PZ - peripheral zone; AFS - anterior fibromuscular stroma; CZ - central zone; U - urethra; ED ejaculatory ducts (1)

Courtesy: Borgis - Post'py Nauk Medycznych 11/2016, s. 812-818 | DOI:

The transitional zone on MR imaging, is found anterior and lateral to the prostatic urethra and constitutes the remaining $5 \%$ of the glandular prostate.

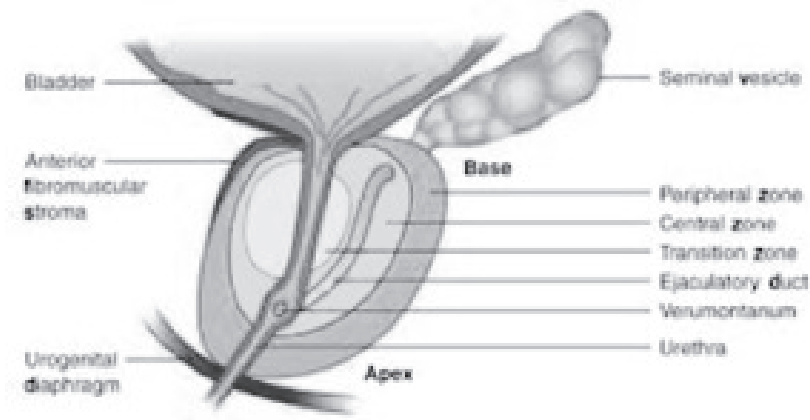

Fig. 2 Schematic shown in sagit tal view of prostate illustrates normal zonal description of prostatic anatomy. Courtesy: Sadhna Verma andArumugam Rajesh AJR:196, March 2011

In benign prostatic hyperplasia (BPH), transitional zones grow disproportionately and eventually surpass the volume of the peripheral zone[3].

Indications and contraindications for prostate biopsies

The indications for prostate biopsy include suspicion of prostate cancer, abnormal digital rectal exam, elevated serum PSA, or PSA velocity. The screening guidelines are available from the National Comprehensive Cancer Network (NCCN)guidelines for early cancer diagnosis[14]. The benefit of PSA screening is higher in African-Americans, patients with a positive family history, and patients taking 5-alpha reductase inhibitors. Elevated serum PSA is nonspecific test; in addition to prostate cancer, prostatitis and BPH can cause the PSA level to be elevated[15]. The recommendation of American Cancer Society is that average risk men expected to live at least ten more years should discuss screening for prostate cancer at age 50 with their physician[16]. These recommendations may be difficult to interpret or to translate into real-life guidance. The benefits of PSA screening must be weighed against the risks of overdiagnosis.

Usually the threshold for prostate-specific antigen (PSA) is $4.0 \mathrm{ng} / \mathrm{mL}$. I t was suggested to lower the normal threshold of PSA to $2.5 \mathrm{ng} / \mathrm{mL}$, but doubles the number of men defined as abnormal and does not clearly result in a benefit[17]. The use of PSA velocity is also controversial. A study demonstrated an association between prostate cancer detection and PSA velocity $>0.75 \mathrm{ng} / \mathrm{mL} / \mathrm{year}[18]$, however, the other data did not find that PSA velocity improved detection[19].

Relative contraindications to prostate biopsy include coagulopathy, painful anorectal conditions, significant immunosuppression, acute prostatitis.

The prophylactic antibiotics prior to transrectal prostate biopsies to reduce infection risk is recommended by the American Urological Association Best Practice Policy Statement on Urological Surgery Antimicrobial Prophylaxis guidelines[20]. There is no single standard protocol, and practice patterns vary widely, often by geography and relative levels of bacterial antibiotic resistance in the community. Fluoroquinolones demonstrate excellent tissue penetration in the prostate. Alternatives to ciprofloxacin include aminoglycosides with metronidazole or clindamycin. However, fluoro quinolones are generally preferred due to their broad-spectrum coverage against Escherichia coli, the most likely infectious organism after biopsy[20]. Quinolone resistance is responsible for the majority of infectious complications after prostate biopsy, with overall rates varying between $<1 \%$ and $5 \%$ [21]. If patients present with post prostate biopsy symptoms, empirical treatment with ceftriaxone, ceftazidime, or amikacin is indicated[22]. No definite evidence for the 
superiority of a longer course of antibiotics (three days vs. one day) or multiple-dose treatment over single dosing[23]. Mechanical bowl cleansing with selfadministered enema prior to biopsy is common, but the value of doing so is under debate[24].

\section{Nerve block}

Topical lidocaine gel can also be used to reduce the discomfort of ultrasound probe insertion and needle puncture. Since the patient is awake, the nerve block can be repeated if inadequate.

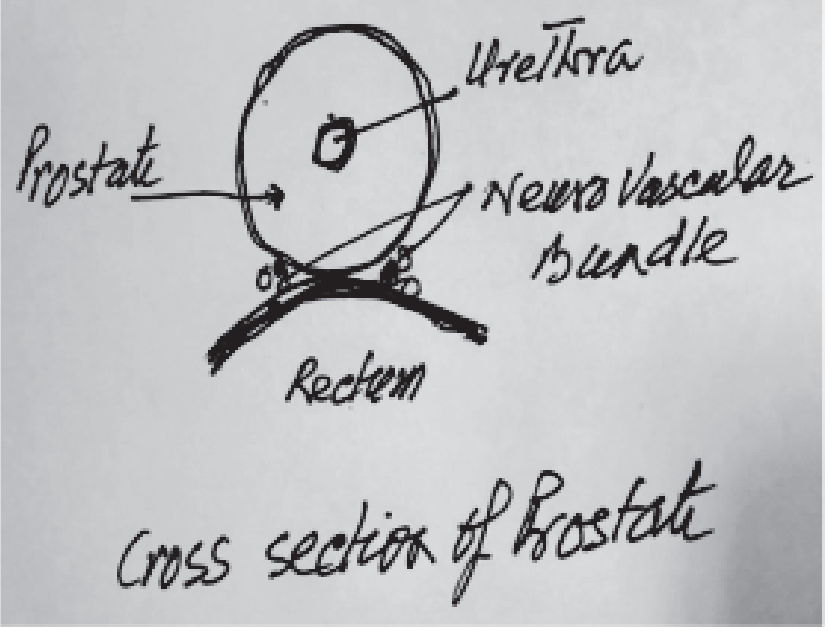

Fig.-3: The neurovascular bundles (NVB)

Bilateral nerve blockade facilitates the procedure immensely by helping to keep the patient immobile, and markedly improving patient comfort for an otherwise uncomfortable procedure. Blockade can also be done quickly with ultrasound guided intra prostatic block. The author's personal preference and practice goes with a single injection $10 \mathrm{ml} 2 \%$ lignocaine directly in to the prostate tageting the prostate just beneath the prostatic capsule with transrectal probe. This simple technique is fast and equally effective compared to periprostatic nerve Block prior to TRUS guided Biopsy[20].

\section{Standard 12 core TRUS biopsy}

Prostate cancer detection is still an area currently fraught with many unanswered questions and much controversy. The optimal number of biopsies needed to identify all patients with prostate cancer at the earliest stage possible for optimal treatment, outcome, and survival is still not known.

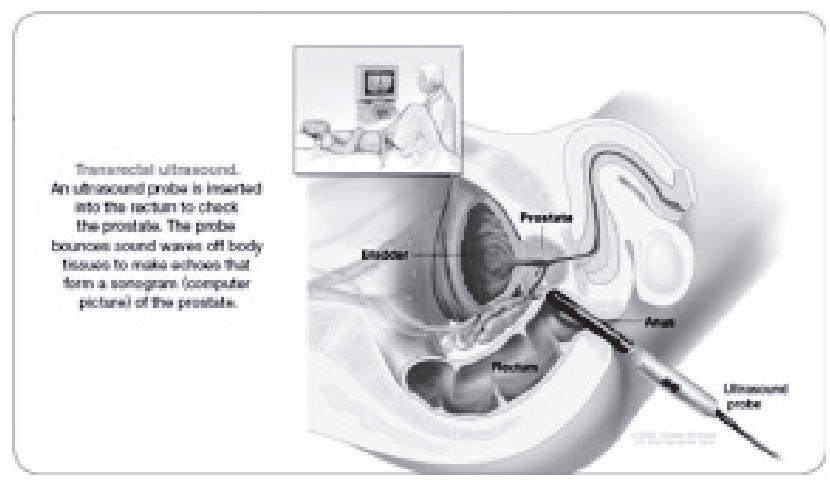

Fig.4. Principle of TRUS guided Prostate Biopsy

Courtesy: William Faloon editorial, May 2013 issue of Life Extension magazine ${ }^{\circledR}$

Generally the patient is positioned in the left lateral decubitus position with knees to the chest and flexed hips on the table edge, allowing mobility of the ultrasound transducer. Core biopsies are obtained using a disposable spring-loaded biopsy needle inserted parallel to the end-fire ultrasound probe through an attached disposable transrectal needle guide. The conventional biopsy consists of twelve cores: the base, middle, and apex sextant regions are sampled in both the lateral and medial aspects of each sextant region on both side.

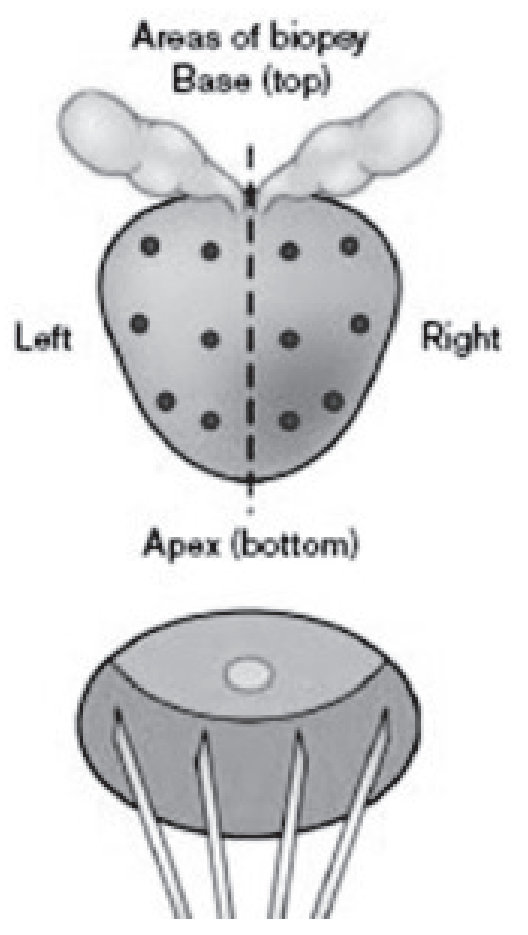

Fig.-5: This diagram depicts a 12-core needle biopsy of a prostate gland. (Yavuz 2008; Redman 2008). 
A standard TRUS guided biopsy begins with a brief survey of the prostate gland to identify nodules, with digital rectal examination and TRUS. The presence of a nodule, requires a through scanning in two planes, typically the axial and sagittal planes. This can be accomplished by rotating the probe slowly while maintaining the nodule in view. On sagittal imaging, imaging is typically in an extreme lateral plane because the image at this plane is composed almost entirely of peripheral zone.

End-fire probes have curved array detectors on the probe tip while side-fire ultrasound probes have longitudinal transducers. End-fire trans rectal ultrasound probes are reported to have higher cancer detection rates compared to side-fire ultrasound probes $[25,26]$ which are limited to a longitudinal biopsy trajectory. End-fire probes allow better sampling in lateral and anterior aspects of prostatic tissue which are typically under sampled[26]. However, in one prospective study involving experienced urologists, ultrasound probe configurations did not differ in cancer detection rates[27].

Manipulations with TRUS should be performed in one plane at a time for better control over the planning of the needle pathway with real time imaging feedback. And it is easier to retrace steps to re-target the biopsy if a systematic approach is used.

\section{Saturation biopsy}

Saturation biopsy refers to obtaining many cores (often 20 or above) throughout the prostate aiming to sample virtually all of the tissue at regular intervals. The saturation biopsy is typically reserved for patients with previous negative biopsies that continue to have a high degree of clinical suspicion for prostate cancer[11]. Risks of missing significant cancer should be balanced with the possibility of detecting a clinically unimportant cancer. It is interesting to note that, the increased number of cores does not appear to be associated with a detectable increased risk of complications[11]. However, the high cost and requirement for regional or a general anesthesia in the hospital setting make it reserved for indicated cases.

\section{Fine needle aspiration}

Most prostate biopsies are obtained as core samples. Although controversial, some studies have shown that fine needle aspiration (FNA) biopsy of the prostate gland may be as effective as core biopsies in cancer detection, but not in characterization or scoring[28].
However, since Gleason scoring is such an important part of prostate cancer management, it is unlikely that FNA will replace core biopsies.

\section{Transperineal biopsy}

Most of the prostate biopsies are obtained transrectally, still there are reasons to consider the transperineal approach. Anatomically, the transperineal approach may identify proportionally more anterior tumors. Moreover, biopsy does not entail crossing the rectal mucosa with presumably lower rates of infections[29,30]. Detection rates, cancer core rates, and complications are generally comparable between the transperineal and transrectal approach[30]. The transperineal biopsy may be best used for patients who do not have rectal access due to previous surgery.

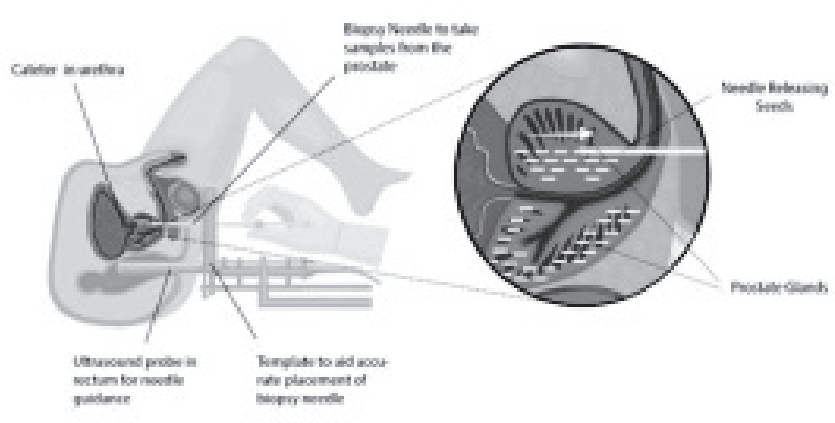

Fig.-6. Principles of Transpereneal Biopsy. Courtesy :Ben Ong November 1, 2017 http://www.bensprostate.com

The transperineal biopsies may require spinal or general anesthesia, or deep sedation, limiting its usage in the office setting. However, this approach may be useful in guiding biopsies under MRI. A brachytherapy grid or stepper, which is a plastic block with pre-drilled holes is used to direct the needles to the proper location. A robotic or semiautomatic needle guide devices are also available for use with MRI guidance via the transperineal approach.

This approaches may also prove useful for focal laser ablation or cryo-ablation or hemi ablation of Prostate. When thermal energy methods (e.g. laser ablation) are utilized, MR guidance can provide real time thermometry of prostate.

A template-assisted prostate biopsy (saturation biopsy), recently proposed as initial biopsy, might have the merit of taking cores more evenly from the whole prostate and the outcome might not be too influenced by the skill of the operators. 
The concept of extended biopsies has been equally applied to the transperineal approach, which has proved to provide the same results as those achieved with the transrectal one.

\section{Complications of prostate Biopsy}

The TRUS biopsy is generally considered to be safe. When a transrectal prostate biopsy is obtained, fecal matter may be introduced into the prostate and presents an infection risk.

Over the five-year study period, 71 patients required admission to Cabrini Hospital for sepsis following TRUS biopsy and were matched to 219 controls. The mean proportion of patients with sepsis across the five-year study period was $1.5 \%$, with the lowest rate of $0.6 \%$ reported in 2011 and the highest rate of $2.9 \%$ reported in 2013. No statistically significant difference regarding the rate of sepsis over the study period was noted ( $p$ $=0.091)[56]$.

In another study the risk of sepsis is estimated about $<0.1 \%$ and the risk of rectal bleeding is $2.1 \%$. Mild hematuria and hematospermia are not uncommon. The morbidity of repeat biopsy does not differ significantly from the initial biopsy[31]. The hematospermia is typically not clinically meaningful but may last many weeks, it should be discussed to avoid anxiety. Significant bleeding, dizziness, or fever should be reported immediately. In a study, the overall risk of hospitalization within 30 days of a prostate biopsy $(6.9 \%)$ and was significantly higher than randomly selected controls (2.7\%) [32]. While it is plausible that a self-administered enema may reduce the fecal matter present, it has not been shown to decrease complication rates and serious infections can occur not withstanding pre-procedure enemas[24].

\section{Doppler and Elastography}

The term "Color Doppler" refers to the peak Doppler shift measured by the probe. The term "Power Doppler", which is also displayed in color, refers to the area under the Doppler curve and tends to have better signal-tonoise ratios. However, Power Doppler is not markedly more accurate than that of Color Doppler[35]. Color and Power Doppler are based on the frequency shift caused by the movement of specular reflectors (the red blood cells) relative to the ultrasound probe. Thus, detection of the Doppler shift can show the direction and speed of blood flow. Flow is usually minimal and symmetric in the normal prostate gland, but color doppler signal may be seen in neurovascular bundles and pericapsular and periurethral arteries as well as a web of peri-prostatic veins[1]. The suspicious areas of focal or asymmetric hyper vascularity within the gland are more likely to demonstrate malignancy. However, the finding is non-specific, as some tumors are hypovascular whereas some benign lesions, particularly prostatitis, are hypervascular[33]. The use of Color Doppler in prostate biopsy can improve sensitivity, but the effect on specificity is not as pronounced[34].

Most tumors has increased blood flow which can be seen with ultrasound contrast agents. Ultrasound contrast agents are microbubbles with a thin membrane containing gas. A large retrospective study, the perpatient detection rate of contrast-enhanced Doppler ultrasound targeted biopsy was $27 \%$, compared to $23 \%$ with systematic biopsy, with a detection rate of $31 \%$ when both modalities were combined (36). When contrast enhanced Doppler targeted biopsy was positive, significantly higher Gleason scores were found[37].

The ultrasound elastography quantifies the stiffness of tissue during the manual compression of the gland by the transducer[1]. Tumors typically have increased stiffness compared to the surrounding tissue. Targeted sono elastography may improve cancer detection rate compared to systematic biopsy. Even with fewer cores obtained, by this technique, having a significantly higher cancer detection rate per core[38]. Although the use of these techniques for targeted biopsy can potentially improve prostate cancer detection rates, limitations include the requirement for technical expertise, lack of updated equipment, subjective interpretive criteria, and inter-user variability.

\section{MRI as diagnostic tool}

MRI has superior soft tissue resolution and better visualization of surrounding anatomy compared to conventional ultrasound. Classical prostate MRI involves placement of an endo-rectal coil in the patient's rectum to obtain higher signal. Although a body or surface coil can be used instead, it reduces sensitivity and specificity of MRI. Modern 3T MRI scanners can obtain excellent quality imaging of the prostate with only multi-channel phased array surface coils. MRI relies on multiple parameters to achieve its accuracy. Multiparametric imaging ( $\mathrm{mpMRI}$ ) consists of a combination of T2-weighted imaging, Diffusion-weighted imaging (which generate apparent diffusion coefficients maps), MR spectroscopy, and Dynamic-contrast enhanced MRI. More of these parameters turn positive, a lesion can be assigned a higher suspicion level[ 39,40$]$. 
Multiparametric MRI may predictive of tumor aggressiveness or grade. On T2-weighted imaging, prostate cancers typically demonstrate lower signal intensity in comparison to the high signal intensity of normal prostate tissue, especially in the peripheral zone where most cancers reside[41]. The Gleason score and D'Amico clinical risk scores are also negatively correlated with the apparent diffusion coefficients derived from diffusion-weighted MRI $[9,42]$.

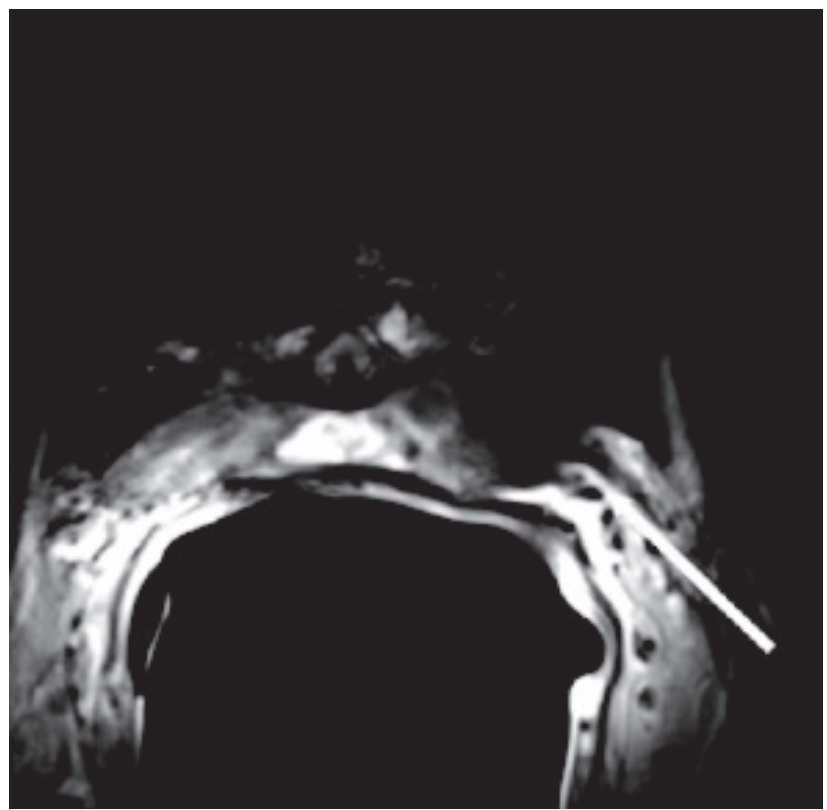

Fig.-7: Extracapsular extension of tumor. A 64-yearold male with biopsy-confirmed Gleason $7(3+4)$ prostate carcinoma. Axial T2-weighted image obtained with the endorectal coil shows the low signal tumor in the left peripheral zone with minimal extension along the left neurovascular bundle (arrow) Courtsey: Sangeet Ghai and Masoom A. Haider ${ }^{1}$ Multiparametric-MRI in diagnosis of prostate cancer Indian J Urol. 2015 Jul-Sep; 31(3): 194-201.

The mpMRI correlates with both the D'Amico risk stratification in visible lesions in the prostate and the cancer detection rate[39,43,40]. In addition, mpMRI can be used to detect clinically significant anterior tumors, which may be missed on TRUS biopsy[44]. mpMRI has been shown to improve the classification accuracy when used with standard clinical criteria for patient selection for active surveillance when compared to radical prostatectomy pathology[45]. MRI can be used to accurately estimate tumor volumes (46), and in one study lesions localized on imaging had a $98 \%$ positive predictive value for prostate cancer as compared to whole gland histology, with greater sensitivity for higher grade tumors[47].

Adapted from breast imaging a "Prostate Imaging Reporting and Data System" (PI-RADS) was published by the European Society of Urogenital Radiology (ESUR): PI-RADS ${ }^{\mathrm{TM}}$ version1. This first guideline paper was based on a summary score for each lesion assessed in different sequences of $\mathrm{mpMRI}$, consisting of T2w, DWI and DCE-MRI and spectroscopy facultatively. These guidelines have been updated recently by a steering committee including the American College of Radiology (ACR), ESUR and the AdMeTech Foundation to the PI-RADS ${ }^{\mathrm{TM}}$ V2. In this version spectroscopy was omitted and DCE-MRI was attributed a minor role. In contrast to version 1 each lesion is attributed a single score based on findings of mpMRI. The objectives of these guidelines were to promote global standardization of prostate imaging, to improve detection, localization, characterization, risk stratification of prostate cancer in treatment naïve prostate as well as to improve communication with referring urologists[48]. The latest PI-RADS version assesses the likelihood (probability) of clinically significant prostate cancer on a 5-point scale for each lesion as follows:

- PI-RADS 1 - Very low (clinically significant cancer is highly unlikely to be present)

- PI-RADS 2 - Low (clinically significant cancer is unlikely to be present)

- PI-RADS 3 - Intermediate (the presence of clinically significant cancer is equivocal)

- PI-RADS 4 - High (clinically significant cancer is likely to be present)

- PI-RADS 5-Very high (clinically significant cancer is highly likely to be present)

\section{MRI-guided biopsies}

The areas suspicious for tumor may be visualized on MRI, it can also be used to guide prostate biopsy (49). This may be particularly important in the setting of anterior or central lesions as systematic biopsy only targets the lateral peripheral zone. MRI guidance has been performed in both open and closed-bore MR systems. While open systems allow easy access to the patient, closed-bore systems offer much higher signal-to-noise ratios and thus, clearer prostate cancer visualization[3]. Most in-gantry MRI guided biopsy studies have used a transrectal approach with a closed- 
bore $3 T$ MR imaging system and an endorectal applicator[3].

Diagnostic multiparametric MR imaging is performed prior to the biopsy for planning purposes[50]. The needle guide may be filled with gadolinium-based contrast material for visualization on MR imaging, and there are commercial automated or robotic systems for transrectal and transperineal access. However, all devices must be MR-compatible, and the patient must be in the MRI gantry throughout the procedure, often in the uncomfortable prone position. Since they may occupy up to four slots on an MRI schedule, they are costly and burdensome on the limited resource of MRI. Most urologists may have limited access to an MRI suite and may not be cost effective. MRI/US fusion has thus been developed to address these issues[51].

\section{MRI/US fusion biopsies}

The MRI/Ultrasound fusion guided biopsy combines a specialized magnetic resonance imaging (MRI) scan with an ultrasound image to help urologists precisely target the area of the prostate that needs to be biopsied. MRI/US fusion superimposes pre-procedural diagnostic MRI images over a real-time ultrasound obtained at a different time and place.

This allows targeting of suspicious lesions seen on MRI to be biopsied under real time ultrasound. The goal is to combine the high soft tissue resolution of the MR image with the real-time visualization of TRUS, in a more comfortable office setting without requiring the physical presence of the MRI gantry. The operator can guide the biopsy needle to specific locations after coregistering the imaging using electromagnetic sensors, which allow the system to determine the spatial position of the ultrasound probe.

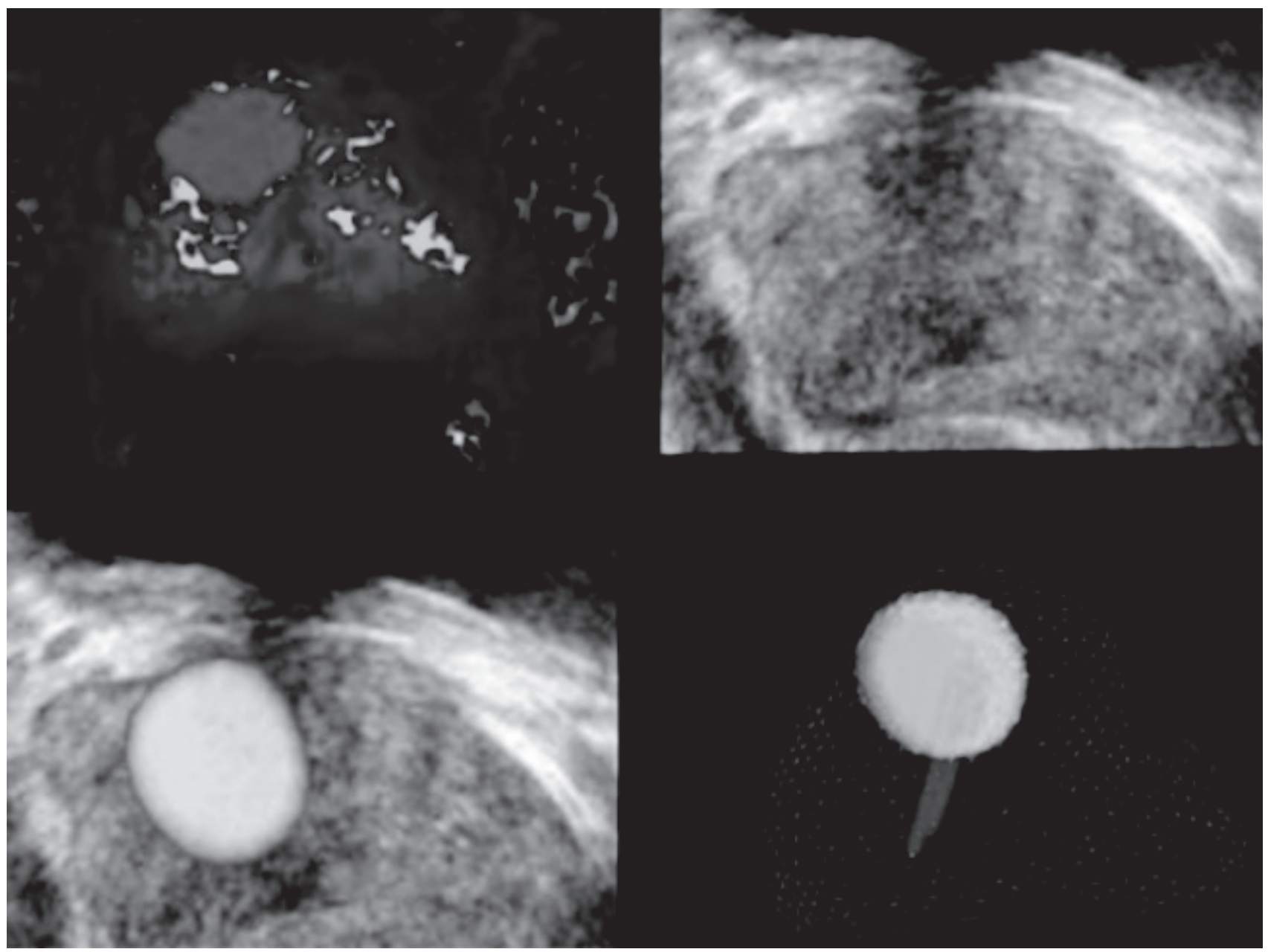

Fig. 8: The MRI/Ultrasound fusion guided biopsy of prostate , A) MRI shows an area suspicious for cancer (red *). (B) That area is invisible to ultrasound. (C) When the MRI and ultrasound are blended, however, the suspicious area shown by MRI becomes evident on the ultrasound (yellow). (D) During the biopsy, the urologist can confirm that the needle reached the suspicious region.. Credit: Image courtesy of UT Southwestern Medical Center 
The registration process requires that a volumetric ultrasound image. Volumetric data can be obtained by using a 3D ultrasound probe .

\section{MRI cognitive biopsy}

MRI Cognitive biopsy is fast and free, but requires expertise and rigorous technique. If these conditions are met, the results are satisfactory. However, the accuracy of cognitive biopsy is probably slightly inferior to that of MRI - fusion biopsy. While some have attempted to perform "cognitive fusion", i.e. use the human brain to estimate the location of a lesion on TRUS, results are better with a standardized fusion approach. Recent studies have shown that MRI/US fusion significantly increases the per-core and perpatient cancer detection rates[12]. IBy targeting the suspicious lesions with this platform, it is possible to selectively sample areas more likely to contain malignancy and preferentially detect higher grade tumors more likely to warrant intervention. However, as a relatively new technique, long term outcomes of MRI/US fusion biopsy are not well studied.

\section{MRI vs. Biopsy}

A recent study by a Bangladeshi Scientist urologist Hashim U Ahmed and and his group concluded that, TRUS-biopsy performs poorly as a diagnostic test for clinically significant prostate cancer. MP-MRI, used as a triage test before first prostate biopsy, could identify a quarter of men who might safely avoid an unnecessary biopsy and might improve the detection of clinically significant cancer[58]. It also suggests that $\mathrm{MRI}$ is twice as likely to spot prostate cancer. A group of researchers at the University College London led the Prostate MRI Imaging Study (Promis), and showed that MRI can pick up $93 \%$ of aggressive prostate cancer cells.

Unlike prostate biopsy that tends to miss cancer, MRI scans can easily detect it in a non-invasive way. The research team was also able to use MRI to see if the tumor is aggressive or slow-growing.

And because it has become much easier to identify tumors that are not life-threatening, it's much easier to determine if treatment is needed or not. The study was also able to reduce cases of overtreatment by $5 \%$. Lead author Hashim Ahmed says that unlike MRI, a prostate biopsy does miss cancer because tissue samples are taken at random. Though it can detect its presence, it cannot confirm if the cancer is aggressive or not[58].

The findings are a breakthrough and could bring about change of practice in prostate cancer detection and treatment. Prostate Cancer UK helped fund the research, and is now working on bringing MRI scans into prostate cancer treatment facilities. It's only a matter of time before the practice of using MRI to detect prostate cancer becomes universal[58].

\section{Negative biopsy}

A negative prostate biopsy does not definitively exclude the presence of cancer. Men who have had one negative biopsy may still have prostate cancer. Patients with negative prostate biopsy should be carefully followed up for raised PSA, abnormal digital rectal examination (DRE), free PSA to total PSA expressed as a percentage (free-to-total PSA\%), PSA density and PSA velocity, novel biomarkers, such as the prostate cancer gene 3 (PCA3) may also be assessed.

There is a trend towards the use of adjuncts to improve the cancer detection yield following a negative first transrectal ultrasound-guided (TRUS) biopsy. Sampling strategies and imaging techniques currently under investigation for improving prostate cancer diagnosis rates include: repeat TRUS biopsy following multiparametric MRI. A contrast-enhanced ultrasound and biopsy, elastography and biopsy can be performed.

Most of these techniques have been introduced at a local level based on facilities available. The majority of tumors are known to be in the posterior zone of the prostate, but tumors that occur in the anterior zone of the prostate are often missed with TRUS biopsies, particularly in large prostates. Sampling this area is improved with template (perineal) biopsies or with saturation biopsies. Multiparametric MRI localises the lesion(s) of interest in the prostate to permit more accurate placement of the biopsy needle. Template biopsies cannot be performed under local anaesthesia, so there are cost implications.

In a study of sequential systematic biopsies, the detection rates of the first, second, third, and fourth biopsy were $22 \%, 10 \%, 5 \%$, and $4 \%$ respectively, and the third and fourth biopsy attempts had slightly higher complication rates and detected lower grade tumors[55]. In a cohort of 195 men with prior negative biopsies, $37 \%$ of subjects were found to have cancer using a combination of TRUSguided and MR/US fusion biopsy[53].

\section{Do Biopsies Risk Spreading Cancer Cells?}

Studies reveal that new tumors are formed in "tracks" or the path within the prostate gland where biopsy needles are inserted. Some experts believe that the 
new tumors are implanted when tumors extracted by biopsy needles are "seeded" along the path of insertion.

A 2014 study provides evidence for this. The research team reviewed related papers about the incidence of seeding and clinical findings. They also looked at some risk factors like the type of needle used, TRUS guided biopsy vs transperineal biopsy, and the cancer's grade and stage[57]. The study reveals that 26 published papers reported 42 cases of track seeding.

Though critics acknowledge the results as valid, they think that the fear caused by the news is overblown. They emphasize that cases of track seeding are very rare. And that there's almost no evidence to suggest that the spread of cancer cells through biopsy led to a "true spread" of prostate cancer.

Yet the findings are enough to compel us to think twice about the safety of the procedure. Over the past 20 years, there has been a massive increase in the number of prostate biopsies done each year. In 2013, about 2 million prostate biopsies were carried out in the US. Think about these numbers.

There is no doubt that biopsies risk spreading cancer cells. Though it is the standard way to detect prostate cancer, Indeed, there are cases in which prostate cancer is slow-growing and non-aggressive, and it wouldn't make a difference if a prostate biopsy will confirm it or not.

\section{Alternatives To Prostate Biopsies}

Doctors have long relied on biopsies to determine prostate cancer. And biopsies are proven to be dangerous. Often, a prostate biopsy finds traces of lowgrade cancer that don't need to be treated.

\section{MRI}

But in recent years, doctors have found out that new imaging studies like high-resolution MRI and ultrasound can be alternatives to prostate biopsy.

They have proven to be very accurate and safe. A multiparametric MRI or a color Doppler ultrasound has a 95 to $98 \%$ chance of determining the location and grade of prostate cancer cells.

According to the Diagnostic Center for Disease, an MRI scan predicts and confirms the presence of prostate cancer more frequently than a biopsy. A hi-res MRI features the most sensitive and specific imaging modality that allows it to produce a very clear picture of the entire prostate and pelvic region.
Experts claim that this is a quantum leap from the blind biopsy approach. It will minimize the need for biopsies, and could potentially save millions of dollars for the healthcare industry.

\section{PCA3 Test}

The PCA3 test is another alternative. PCA3 stands for "Prostate Cancer gene 3", a protein that is produced by prostate cancer cells at much higher levels. PCA3 leaks into the urine when the prostate gland is stimulated. Unlike the PSA test, a high PCA3 test can only result from cancer - not from an enlarged prostate, inflamed prostate or other non-cancerous prostate problem. For this test, you need to undergo a DRE. This will stimulate PCA3 to leak into the urine. A urine sample is then collected and sent to the lab. It takes about 1-2 weeks to get the results. The higher the PCA3 score, the more likely you have prostate cancer. This test is also used to determine the effectiveness of cancer treatment. The higher the score, the more aggressive is the prostate cancer. Prostate cancers are often slow-growing and non-aggressive. It wouldn't matter if a non-aggressive cancer is detected or not, and it doesn't need any treatment.

And even if you have an aggressive prostate cancer, there are alternatives to a prostate biopsy, like MRI and the PCA3 test. These methods are non-invasive and accurate. Plus, they don't have the negative side effects that result from a prostate biopsy.

\section{4Kscore Prostate Cancer Test}

The 4Kscore Test combines four prostate-specific kallikrein assay results with clinical information in an algorithm that calculates the individual patient's percent risk for aggressive prostate cancer. It is the most accurate test to assess a patient's risk for aggressive prostate cancer prior to a prostate biopsy. With the 4Kscore Test, physicians can more confidently choose to place a low-risk patient under active monitoring or perform a biopsy on a high-risk patient based on their clinical evaluation, enhanced by the 4Kscore Test result.

The 4Kscore Test has undergone extensive clinical development and confirmation. The biomarkers utilized in the 4Kscore Test are based on over a decade of research conducted by scientists at the Memorial Sloan Kettering Cancer Center and leading research centers in Europe, encompassing over 20,000 men in Europe and the United States. The results have also been replicated in a prospective, blinded clinical study conducted at 26 urology centers across the United States on 1,012 patients. 
The test has been shown to identify the actual risk of aggressive prostate cancer for the individual patient, including high grade prostate cancer pathology and poor prostate cancer clinical outcomes within 20 years, with both high sensitivity and negative predictive value for aggressive prostate cancer.

Voigt and coworkers[1] performed a cost-analysis on a theoretical group of 100,000 men. They were evaluated with conventional management, in which all the men underwent biopsy and then appropriate men were treated. Also included were the costs of managing complications. The investigators compared those costs with those of men who had the $4 \mathrm{~K}$ test and only underwent biopsy if the value was $7.5 \%$ or greater. Any $4 \mathrm{~K}$ values that were lower were assumed to be managed conservatively. This is based on the finding that over 20 years, men with $4 \mathrm{~K}$ scores lower than $7.5 \%$ have been found to progress to advanced prostate cancer only rarely.

The results are very important. By comparing the two approaches, the analysis found that about $64 \%$ of the men could avoid a prostate biopsy. Thirty-nine percent of the men who would have been diagnosed and treated with Gleason 6 cancer were able to avoid treatment. This process resulted in less than $2 \%$ of significant cancers being missed and no Gleason 8 cancers being missed. The $4 \mathrm{~K}$ test had a high likelihood of finding the threatening cancers without missing many of them. The cost savings would be about $16 \%$ overall, including the costs of diagnosis, treatment, managing any complications, and also follow-up of those men who had a negative biopsy or did not have a biopsy[59].

This is an important piece of information in an interesting and well-done analysis. The results make a very strong case for incorporating the $4 \mathrm{~K}$ test into standard management of men who have been advised to undergo a prostate biopsy because of PSA test results. Time will tell how rapidly this is incorporated into routine care, but on the basis of this report, I believe one can make a very strong argument that the $4 \mathrm{~K}$ test should be used on a routine basis $[58,59]$.

\section{Conclusion}

The best procedure biopsy is still evolving. The principle and the techniques will leverage the skill sets of both urologists and radiologists in a collaborative team approach. The use of real-time navigation systems for spatial cancer mapping could improve the diagnostic yield of biopsy and could play a major role in the screening, evaluation, diagnosis, surveillance and management of the prostate cancer patient.

\section{References}

1. Borkowetz A, Hadaschik B, Platzek I et al. Prospective comparison of transperineal magnetic resonance imaging/ultrasonography fusion biopsy and transrectal systematic biopsy in biopsy-naive patients. BJU Int 2018;121: 53-60

2. Siddiqui MM, Rais-Bahrami S, Turkbey B et al. Comparison of MR/ultrasound fusion-guided biopsy with ultrasound-guided biopsy for the diagnosis of prostate cancer. JAMA2015; 313: 390-7

3. Pokorny MR, de Rooij M, Duncan E et al. Prospective study of diagnostic accuracy comparing prostate cancer detection by transrectal ultrasound-guided biopsy versus magnetic resonance (MR) imaging with subsequent MR-guided biopsy in men without previous prostate biopsies. Eur Urol 2014; 66: 22 9

4. Schröder FH, Hugosson J, Roobol MJ, et al. Screening and prostate-cancer mortality in a randomized European study. N Engl J Med. 2009;360:1320-1328. [PubMed]

5. Andriole GL, Crawford ED, Grubb RL, 3rd, et al. Mortality results from a randomized prostatecancer screening trial. $\mathrm{N}$ Engl J Med. 2009;360:1310-1319. [PMC free article] [PubMed]

6. Ilic D, Neuberger MM, Djulbegovic M, Dahm P. Screening for prostate cancer. Cochrane Database Syst Rev. 2013;1:CD004720. [PubMed]

7. Lin K, Lipsitz R, Janakiraman S. Preventive Services Task Force [Internet] Rockville (MD): Agency for Healthcare Research and Quality (US); 2008. [cited 2013 Sep 1]. Benefits and Harms of Prostate-Specific Antigen Screening for Prostate Cancer: An Evidence Update for the U.S. Available from: http://www.ncbi.nlm.nih.gov/books/ NBK43401/ [PubMed]

8. Hodge KK, McNeal JE, Terris MK, Stamey TA. Random systematic versus directed ultrasound guided transrectal core biopsies of the prostate. J Urol. 1989;142:71-74. discussion 74-75. [PubMed]

9. Nelson ED, Slotoroff CB, Gomella LG, Halpern EJ. Targeted biopsy of the prostate: the impact of 
color Doppler imaging and elastography on prostate cancer detection and Gleason score. Urology. 2007;70:1136-1140. [PubMed]

10. Berger AP, Gozzi C, Steiner H, et al. Complication rate of transrectal ultrasound guided prostate biopsy: a comparison among 3 protocols with, 6 , 10 and 15 cores. J Urol. 2004;171:1478-1480. discussion 1480-1481. [PubMed]

11. Jones JS, Patel A, Schoenfield L, Rabets JC, Zippe CD, Magi-Galluzzi C. Saturation technique does not improve cancer detection as an initial prostate biopsy strategy. J Urol. 2006;175:485488. [PubMed]

12. Pinto PA, Chung PH, Rastinehad AR, et al. Magnetic resonance imaging/ultrasound fusion guided prostate biopsy improves cancer detection following transrectal ultrasound biopsy and correlates with multiparametric magnetic resonance imaging. J Urol. 2011;186:1281-1285. [PMC free article] [PubMed]

13. McNeal JE. The zonal anatomy of the prostate. The Prostate. 1981;2:35-49. [PubMed]

14. NCCN Clinical Practice Guidelines in Oncology: Prostate Cancer Early Detection v2.2012 [Internet] Available from: http://www.nccn.org/professionals/ physician_gls/pdf/prostate_detection.pdf.

15. Nadler RB, Humphrey PA, Smith DS, Catalona WJ, Ratliff TL. Effect of inflammation and benign prostatic hyperplasia on elevated serum prostate specific antigen levels. J Urol. 1995;154:407-413. [PubMed]

16. Wolf AMD, Wender RC, Etzioni RB, et al. American Cancer Society guideline for the early detection of prostate cancer: update 2010. CA Cancer J Clin. 2010;60:70-98. [PubMed]

17. Welch HG, Schwartz LM, Woloshin S. ProstateSpecific Antigen Levels in the United States: Implications of Various Definitions for Abnormal. JNCI J Natl Cancer Inst. 2005;97:1132-1137. [PubMed]

18. Carter HB, Pearson JD, Metter EJ, et al. Longitudinal evaluation of prostate-specific antigen levels in men with and without prostate disease. JAMA. 1992;267:2215-2220. [PMC free article] [PubMed]
19. Schröder FH, Roobol MJ, van der Kwast TH, Kranse $\mathrm{R}$, Bangma $\mathrm{CH}$. Does PSA velocity predict prostate cancer in pre-screened populations? Eur Urol. 2006;49:460-465. discussion 465. [PubMed]

20. Singh SK ${ }^{1}$, Kumar A, Griwan MS, Sen J , Comparative Evaluation of Periprostatic Nerve Block with and without Intraprostatic Nerve Block in Transrectal Ultrasound-Guided Prostatic Needle Biopsy..Korean J Urol. 2012 Aug;53(8):547-51. doi: 10.4111/kju.2012.53.8.547. Epub 2012 Aug 16.

21. Wagenlehner FME, van Oostrum E, Tenke P, et al. Infective complications after prostate biopsy: outcome of the Global Prevalence Study of Infections in Urology (GPIU) 2010 and 2011, a prospective multinational multicentre prostate biopsy study. Eur Urol. 2013;63:521-527. [PubMed]

22. Feliciano J, Teper E, Ferrandino M, et al. The incidence of fluoroquinolone resistant infections after prostate biopsy-are fluoroquinolones still effective prophylaxis? J Urol. 2008;179:952-955. discussion 955. [PubMed]

23. Zani EL, Clark OAC, Rodrigues Netto N., Jr Antibiotic prophylaxis for transrectal prostate biopsy. Cochrane Database Syst Rev. 2011:CD006576. [PubMed]

24. Zaytoun OM, Anil T, Moussa AS, Jianbo L, Fareed $\mathrm{K}$, Jones JS. Morbidity of prostate biopsy after simplified versus complex preparation protocols: assessment of risk factors. Urology. 2011;77:910914. [PubMed]

25. Paul R, Korzinek C, Necknig U, et al. Influence of transrectal ultrasound probe on prostate cancer detection in transrectal ultrasound-guided sextant biopsy of prostate. Urology. 2004;64:532-536. [PubMed]

26. Ching CB, Moussa AS, Li J, Lane BR, Zippe C, Jones JS. Does transrectal ultrasound probe configuration really matter? End fire versus side fire probe prostate cancer detection rates. J Urol. 2009;181:2077-2082. discussion 2082-2083. [PubMed]

27. Rom M, Pycha A, Wiunig C, et al. Prospective randomized multicenter study comparing prostate cancer detection rates of end-fire and side-fire transrectal ultrasound probe configuration. Urology. 2012;80:15-18. [PubMed] 
28. Maksem JA, Berner A, Bedrossian C. Fine needle aspiration biopsy of the prostate gland. Diagn Cytopathol. 2007;35:778-785. [PubMed]

29. Hossack T, Patel MI, Huo A, et al. Location and pathological characteristics of cancers in radical prostatectomy specimens identified by transperineal biopsy compared to transrectal biopsy. J Urol. 2012;188:781-785. [PubMed]

30. Hara R, Jo Y, Fujii T, et al. Optimal approach for prostate cancer detection as initial biopsy: prospective randomized study comparing transperineal versus transrectal systematic 12core biopsy. Urology. 2008;71:191-195. [PubMed]

31. Djavan B, Waldert M, Zlotta A, et al. Safety and morbidity of first and repeat transrectal ultrasound guided prostate needle biopsies: results of a prospective European prostate cancer detection study. J Urol. 2001;166:856-860. [PubMed]

32. Loeb S, Carter HB, Berndt SI, Ricker W, Schaeffer EM. Complications after prostate biopsy: data from SEER-Medicare. J Urol. 2011;186:18301834. [PubMed]

33. Nelson ED, Slotoroff CB, Gomella LG, Halpern EJ. Targeted biopsy of the prostate: the impact of color Doppler imaging and elastography on prostate cancer detection and Gleason score. Urology. 2007;70:1136-1140. [PubMed]

34. Roy C, Buy X, Lang H, Saussine C, Jacqmin D. Contrast enhanced color Doppler endorectal sonography of prostate: efficiency for detecting peripheral zone tumors and role for biopsy procedure. J Urol. 2003;170:69-72. [PubMed]

35. Halpern EJ, Strup SE. Using gray-scale and color and power Doppler sonography to detect prostatic cancer. Am J Roentgenol. 2000;174:623-627. [PubMed]

36. Mitterberger MJ, Aigner F, Horninger $\mathrm{W}$, et al. Comparative efficiency of contrastenhanced colour Doppler ultrasound targeted versus systematic biopsy for prostate cancer detection. Eur Radiol. 2010;20:2791-2796. [PubMed]

37. Mitterberger M, Pinggera GM, Horninger W, et al. Comparison of contrast enhanced color Doppler targeted biopsy to conventional systematic biopsy: impact on Gleason score. J Urol. 2007;178:464-468. discussion 468. [PubMed]
38. Pallwein L, Mitterberger $M$, Struve $P$, et al. Comparison of sonoelastography guided biopsy with systematic biopsy: impact on prostate cancer detection. Eur Radiol. 2007;17:2278-2285. [PubMed]

39. Turkbey B, Shah VP, Pang Y, et al. Is apparent diffusion coefficient associated with clinical risk scores for prostate cancers that are visible on 3T MR images? Radiology. 2011;258:488-495. [PMC free article] [PubMed]

40. Rais-Bahrami S, Siddiqui MM, Turkbey B, et al. Utility of multiparametric magnetic resonance imaging suspicion levels for detecting prostate cancer. J Urol. 2013;190:1721-1727. [PubMed]

41. Hricak H, Choyke PL, Eberhardt SC, Leibel SA, Scardino PT. Imaging prostate cancer: a multidisciplinary perspective. Radiology. 2007;243:28-53. [PubMed]

42. Kurhanewicz J, Swanson MG, Nelson SJ, Vigneron DB. Combined Magnetic Resonance Imaging and Spectroscopic Imaging Approach to Molecular Imaging of Prostate Cancer. J Magn Reson Imaging. 2002;16:451-463. [PMC free article] [PubMed]

43. Rastinehad AR, Baccala AA, Jr, Chung PH, et al. D'Amico risk stratification correlates with degree of suspicion of prostate cancer on multiparametric magnetic resonance imaging. J Urol. 2011;185:815-820. [PMC free article] [PubMed]

44. Lawrentschuk N, Haider MA, Daljeet N, et al. "Prostatic evasive anterior tumours": the role of magnetic resonance imaging. BJU Int. 2010;105:1231-1236. [PubMed]

45. Turkbey B, Pinto PA, Mani H, et al. Prostate cancer: value of multiparametric MR imaging at $3 \mathrm{~T}$ for detection-histopathologic correlation. Radiology. 2010;255:89-99. [PMC free article] [PubMed]

46. Turkbey B, Mani H, Aras O, et al. Correlation of magnetic resonance imaging tumor volume with histopathology. J Urol. 2012;188:1157-1163. [PMC free article] [PubMed]

47. Turkbey B, Mani H, Shah V, et al. Multiparametric 3T prostate magnetic resonance imaging to detect cancer: histopathological correlation using prostatectomy specimens processed in customized magnetic resonance imaging based 
molds. J Urol. 2011;186:1818-1824. [PMC free article] [PubMed]

48. Philipp Steiger and Harriet C. Thoeny Prostate MRI based on PI-RADS version 2: how we review and report Cancer Imaging, 201616:9 https:// doi.org/10.1186/s40644-016-0068-2@ Steiger and Thoeny. 2016

49. Beyersdorff D, Winkel A, Hamm B, Lenk S, Loening SA, Taupitz M. MR Imaging- guided Prostate Biopsy with a Closed MR Unit at $1.5 \mathrm{~T}$ : Initial Results. Radiology. 2005;234:576-581. [PubMed]

50. Pondman KM, Fütterer $\mathrm{JJ}$, ten Haken $\mathrm{B}$, et al. MR-guided biopsy of the prostate: an overview of techniques and a systematic review. Eur Urol. 2008;54:517-527. [PubMed]

51. Xu S, Kruecker J, Turkbey B, et al. Real-time MRITRUS fusion for guidance of targeted prostate biopsies. ComputAided Surg. 2008;13:255-264. [PMC free article] [PubMed]

52. Walton Diaz A, Hoang AN, Turkbey B, et al. Can magnetic resonance-ultrasound fusion biopsy improve cancer detection in enlarged prostates? J Urol. 2013;190:2020-2025. [PubMed]

53. Vourganti S, Rastinehad A, Yerram NK, et al. Multiparametric magnetic resonance imaging and ultrasound fusion biopsy detect prostate cancer in patients with prior negative transrectal ultrasound biopsies. J Urol. 2012;188:2152-2157. [PMC free article] [PubMed]

54. Siddiqui MM, Rais-Bahrami $\mathrm{S}$, Truong $\mathrm{H}$, et al. Magnetic resonance imaging/ultrasound-fusion biopsy significantly upgrades prostate cancer versus systematic 12-core transrectal ultrasound biopsy. Eur Urol. 2013;64:713-719. [PubMed]

55. Djavan B, Ravery V, Zlotta A, et al. Prospective evaluation of prostate cancer detected on biopsies 1, 2, 3 and 4: when should we stop? J Urol. 2001;166:1679-1683. [PubMed]

56. ElliotAnderson, Olivia Leahy, Allen C. Cheng, and Jeremy Grummet, Risk factors for infection following prostate biopsy - a case control study, BMC Infect Dis. 2015; 15: 580. Published online 2015 Dec 23. doi: 10.1186/s12879-015-1328-7

57. Volanis $D^{1}$, Neal DE, Warren AY, Gnanapragasam VJ. Incidence of needle-tract seeding following prostate biopsy for suspected cancer: a review of the literature. BJU Int. 2015 May;115(5):698-704. doi: 10.1111/bju.12849. Epub 2014 Oct 22.

58. Hashim U Ahmed, Ahmed El-Shater Bosaily, , Louise C Brown, Rhian Gabe, Prof Richard Kaplan, Prof Mahesh K Parmar, DPhil,Yolanda Collaco-Moraes, Katie Ward, Richard G Hindley, Alex Freeman,Alex P Kirkham, Robert Oldroyd, Chris Parker, Mark Emberton, and the PROMIS study, group ${ }^{\dagger}$ Diagnostic accuracy of multiparametric MRI and TRUS biopsy in prostate cancer (PROMIS): a paired validating confirmatory study, The lancet, Volume 389, No. 10071, p815822, 25 February 2017.

59. Voigt JD, Dong Y, Linder V, Zappala S. Use of the 4Kscore test to predict the risk of aggressive prostate cancer prior to prostate biopsy: overall cost savings and improved quality of care to the Us healthcare system. Rev Urol. 2017;19:1-10. 